\title{
Effects of Aramchol in patients with nonalcoholic fatty liver disease (NAFLD). A systematic review and meta-analysis
}

\author{
Adnan Malik ${ }^{1}$, Mahum Nadeem², Waseem Amjad³, Muhammad Imran Malik ${ }^{4}$, Sadia Javaid ${ }^{5}$, Umer Farooq \\ Khadija Naseem ${ }^{7}$, Ahmad Khan ${ }^{8}$ \\ ${ }^{1}$ Saint Joseph's Medical Centre, Stockton, California, United States \\ 2Oklahoma University Medical Centre, Oklahoma City, Oklahoma, United States \\ ${ }^{3}$ Harvard Medical School, Boston, Massachusetts, United States \\ ${ }^{4}$ Airedale General Hospital, West Yorkshire, England \\ ${ }^{5}$ Nishtar Medical University Multan, Pakistan \\ ${ }^{6}$ Macneal Hospital, Berwyn, Illinois, USA \\ ${ }^{7}$ West Virginia Charleston Medical Centre, WV, USA \\ ${ }^{8}$ Case Western Reserve University, Cleveland, Ohio, USA
}

Gastroenterology Rev 2023; 18 (1): 67-75

DOI: https://doi.org/10.5114/pg.2022.113573

Key words: non-alcoholic fatty liver disease, Aramchol, meta-analysis.

Address for correspondence: Adnan Malik MD, MPH, MBA, Saint Joseph's Medical Centre, Stockton, California, United States, phone: +1 (404) 786-8961, e-mail: adnanmalik892@hotmail.com

\begin{abstract}
Introduction: Nonalcoholic fatty liver disease (NAFLD) comprises a wide range of related liver disorders affecting mainly people who drink no or very little alcohol. Aramchol is a new synthetic molecule that has been shown to reduce liver fat content. There is little evidence supporting its efficacy in humans.

Aim: To evaluate the efficacy of Aramchol in patients with NAFLD according to different randomized clinical trials.

Material and methods: We searched PubMed, SCOPUS, Web of Science, and Cochrane Library for relevant clinical trials assessing the use of Aramchol in patients with NAFLD. Risk of bias assessment was performed using Cochrane's risk of bias tool. We included the following outcomes: alanine aminotransferase (ALT), aspartate aminotransferase (AST), alkaline phosphatase (AP), glycated haemoglobin $\left(\mathrm{HbA}_{1 \mathrm{c}}\right)$, total cholesterol (TC), triglyceride (TG), HOMA-IR, and insulin level.

Results: We included 3 clinical trials. We found that the Aramchol group did not show any significant difference from the control group regarding ALT $(M D=3.92(-21.20,29.04), p=0.76), A P(M D=-0.59(-8.85,7.67), p=0.89), \mathrm{HbA}_{1 c}(M D=-0.11$ $(-0.32,0.10), p=0.29), \mathrm{TC}(\mathrm{MD}=14.25(-626,34.77), p=0.17), \mathrm{TG}(\mathrm{MD}=2.29(-39.30,43.87), p=0.91), \mathrm{HOMA}-\mathrm{IR}(\mathrm{MD}=$ $-0.11(-1.58,1.37), p=0.89)$, and insulin levels $(\mathrm{MD}=-0.88(-5.82,4.06), p=0.73)$. AST levels were significantly higher in the Aramchol group ( $M D=11.04$ (4.91, 17.16), $p=0.04)$.

Conclusions: Aramchol was a safe and tolerable drug to be used in patients with NAFLD. However, it was not superior to placebo in reducing the biochemical liver markers.
\end{abstract}

\section{Introduction}

Nonalcoholic fatty liver disease (NAFLD) comprises a wide range of related liver disorders affecting mainly people who drink no or very little alcohol [1, 2]. Recently, it has become the most common cause of chronic liver disease, especially in the obese population of the western world, with a potential further increase in prevalence in the coming years [3-6].
Histologically it shows different stages from simple fat accumulation to liver cirrhosis. The earliest stage of the disease comprises a process of excess fat accumulation, called simple fatty liver or steatosis. The deposited triglycerides form cytoplasmic droplets in hepatocytes. Steatosis is often a self-limited process but may progress to a more advanced stage called non-alcoholic steatohepatitis (NASH). NAFLD occurs mainly in the obese, in whom insulin resistance oc- 
curs. No one knows the exact pathogenesis, only the limited treatment options $[2,7,8]$.

The main pathophysiology of NAFLD is increased free fatty acid (FFA) delivery to the liver, as adipose tissue shows increased insulin resistance $[9,10]$. Also, hyperinsulinaemia and excess carbohydrate intake may lead to an increase of de-novo lipogenesis, which represents a distinct character of NAFLD [11]. Despite the high prevalence of NAFLD, it shows no symptoms and is diagnosed incidentally during follow-up for other medical conditions [8]. Since obesity represents the main risk factor, physicians directed the different management plans towards lifestyle modification and weight reduction. Weight loss shows an improvement of both predisposing factors and liver histology in patients with NAFLD [12]. In addition, bariatric surgery represents an option to treat morbidly obese patients with NAFLD, but there are insufficient data to make it a valid choice [13].

Hence, the need for pharmaceutical treatment has increased, to prevent further development of NAFLD. The use of pharmaceutics mainly tends to target the management of associated diseases with NAFLD, including high blood pressure, blood glucose, and cholesterol levels. The progress in understanding the pathogenesis of NAFLD helps in developing specific, targeted medications.

Aramchol (arachidyl amido cholanoic acid) represents a new synthetic molecule. It results from the conjugation of 2 components: cholic acid (bile acid) and arachidic acid (saturated fatty acid). It inhibits stearoyl coenzyme A desaturase (SCD1). SCD1 represents the key enzyme of fatty acid metabolism in the liver. This leads to a direct anti-steatosis effect by decreasing fat synthesis and increasing fat oxidation, which results in the reduction of liver fat storage $[14,15]$. Many studies found that Aramchol significantly reduced the fat content of the liver in animals with a high-fat diet, improved insulin resistance, and reduce the atherogenic effect [16-19]. Since the discovery of its effect on animals, different clinical trials have investigated its efficacy and safety on liver fat reduction. A clinical trial on NAFLD patients found that a specific dose of Aramchol (300 mg) for 3 months reduced the fat content of the liver by nearly $12.5 \%$ compared to placebo, with no serious drug adverse events [20]. This makes Aramchol a promising drug in the treatment of NAFLD. Recent reviews have discussed the available pharmaceutical options for NAFLD in general, with a lack of systematic reviews on Aramchol. This makes it difficult to formulate comprehensive evidence for the clinical use of Aramchol in NAFLD.

\section{Aim \\ Hence, in this systematic review and meta-analy- sis, we aim to evaluate Aramchol in NAFLD patients,}

regarding its efficacy according to different randomized clinical trials.

\section{Material and methods}

In our systematic review and meta-analysis, we followed the Preferred Reporting Items for Systematic Reviews and Meta-analyses (PRISMA) guidelines [21]. All steps of this study were performed according to Cochrane's handbook of systematic reviews of interventions [22].

\section{Literature search}

We searched four databases: PubMed, SCOPUS, Cochrane CENTRAL, and Web of Science, without any restrictions on time or language. We performed our search using the following search strategy: (aramchol OR arachidyl amido cholanoic acid OR scd1 inhibitor) AND (hepat* OR steato*).

\section{Eligibility criteria}

Results from searching the literature were marked as included if they met the following eligibility criteria: (i) Population: patients with non-alcoholic fatty liver disease, (ii) Intervention: Aramchol with any route of administration. (iii) Comparator: any control. (iv) Outcomes: alanine aminotransferase (ALT), aspartate aminotransferase (AST), alkaline phosphatase (AP), glyvated haemoglobin $\left(\mathrm{HbA}_{1 \mathrm{c}}\right)$, total cholesterol (TC), triglyceride (TG), HOMA-IR, and insulin level. (v) Study design: only randomized clinical trials were included in our study (RCTs). Our exclusion criteria were as follows: (1) non-randomized controlled clinical trials, (2) studies that did not report data or measures for our selected outcomes, (3) single-armed trials, (4) those with no available full-text, or (5) animal studies.

\section{Screening of results}

After retrieving the search results, we exported the data into Endnote X8.0.1 (Build 1044), with the automatic removal of any duplicates. We screened the included articles manually through 2 steps: the first step was the title and abstract screening, and the second step was full-text screening. Two independent authors conducted the screening steps and obtained the fulltext files for all included studies based on our criteria for eligibility criteria. A third author solved any discrepancies.

\section{Data extraction and analysis}

After the screening process was completed, we performed the data-extraction step. We extracted the data into 4 main categories: 1) baseline and demograph- 
ic data of patients in each study, including age, body mass index, male/female ratio, and BMI. 2) Baseline values of ALT, AST, Alkaline phosphatase, HOMA-IR, and $\mathrm{HbA}_{1 \mathrm{c}}$. 3) Baseline values of total cholesterol, HDL, LDL, and insulin. 4) Data for analysis that consisted mainly of our included outcomes: ALT, AST, AP, $\mathrm{HbA}_{1 \mathrm{c}}, \mathrm{TC}, \mathrm{TG}$, HOMA-IR, and Insulin. We also extracted the data about the 7 domains assessing the risk of bias according to Cochrane's risk of bias.

\section{Statistical analysis}

We performed our analysis using Review Manager Software (RevMan 5.4.1) under the inverse variance method. Continuous data were expressed using the mean difference (MD) and standard error, relative to 95\% confidence interval $(\mathrm{Cl})$, while dichotomous outcomes were expressed using the percentage and total. Two main tests indicate inconsistency among studies [23]: the $I^{2}$ test $\left(I^{2}\right)$ and the $p$-value of the $\chi^{2}$ test. The outcomes with $I^{2}>50 \%, p<0.1$ were considered heterogeneous, while outcomes with $l^{2}<50 \%, p>0.1$ were considered homogeneous, according to the Cochrane Handbook [22]. We performed the analysis of homogeneous data under a fixed-effects model, while heterogeneous data were analysed under the random-effects model.

\section{Quality assessment}

We performed the quality assessment of this meta-analysis by using the guidelines of the Grading of Recommendations, Assessment, Development, and Evaluations (GRADE). We included only the controlled trials and excluded the observational evidence. To assess the risk of bias among the included studies, we used Cochrane's risk of bias tool [24]. The tool depends on the following domains for assessment of the risk of bias: 1) proper randomization; 2) blinding allocation of the included patients into each group; 3) blinding of patients only (single-blinding), blinding of both personnel and participants (double-blinding), or not blinding at all; 4) attrition bias; 5) selection bias (outcomes reported matches with that of the protocol or not); 6) awareness of the outcome assessor (whether blinded or not); and 7) other bias. The total risk of bias for the studies was also assessed.

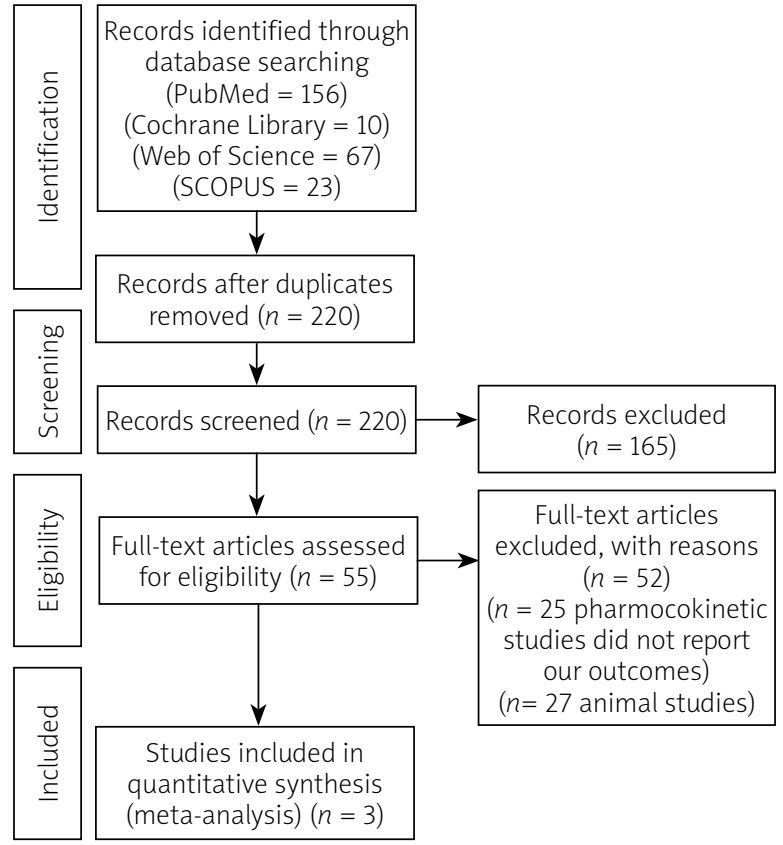

Figure 1. A PRISMA flow diagram of our literature search

\section{Results}

\section{Summary of included studies}

The results of our literature search are described in detail in the PRISMA flow diagram (Figure 1). We present the analysis of 231 patients with NAFLD from 3 studies [20, 25, 26]. A total of 143 patients were allocated to the Aramchol group, while 88 patients were allocated to the control group. The mean age of patients in the Aramchol group and control group was 46.33 years and 48.7 years, respectively. Table I shows a detailed summary of included patients and their demographic data and BMI.

\section{Results of risk of bias assessment}

We found an overall low risk of bias, according to Cochrane's tool [10]. All studies [20, 25, 26] were at low risk of bias regarding randomization, blinding of participants and personnel, attrition, and selective reporting. Concerning allocation concealment, Ajmera et al. [25] reported proper allocation concealment, so it was cate-

Table I. A detailed summary of the included participants and their demographic data

\begin{tabular}{lcccccccc} 
Study & \multicolumn{2}{c}{ Sample size } & \multicolumn{2}{c}{ Age [years] } & \multicolumn{2}{c}{ Sex (male/female) } & BMI [kg/m²] \\
\cline { 2 - 9 } & Aramchol & Control & Aramchol & Control & Aramchol & Control & Aramchol & Control \\
\hline Ajmera 2019 & 25 & 25 & $46.6(11.4)$ & $49.7(9.0)$ & $22 / 3$ & $24 / 1$ & $30.4(3.18)$ & $29.1(2.29)$ \\
\hline Levin 2018 & 101 & 48 & $53.9(10.9)$ & $54.4(10.3)$ & NR & NR & $32.4(4.5)$ & $32.6(4.9)$ \\
\hline Safadi 2014 & 20 & 19 & $38.4(14.6)$ & $42(11.2)$ & NR & NR & $29.1(3.4)$ & $28.2(3.5)$
\end{tabular}

Data represented as mean (SD). BMI - body mass index, NR - not reported. 


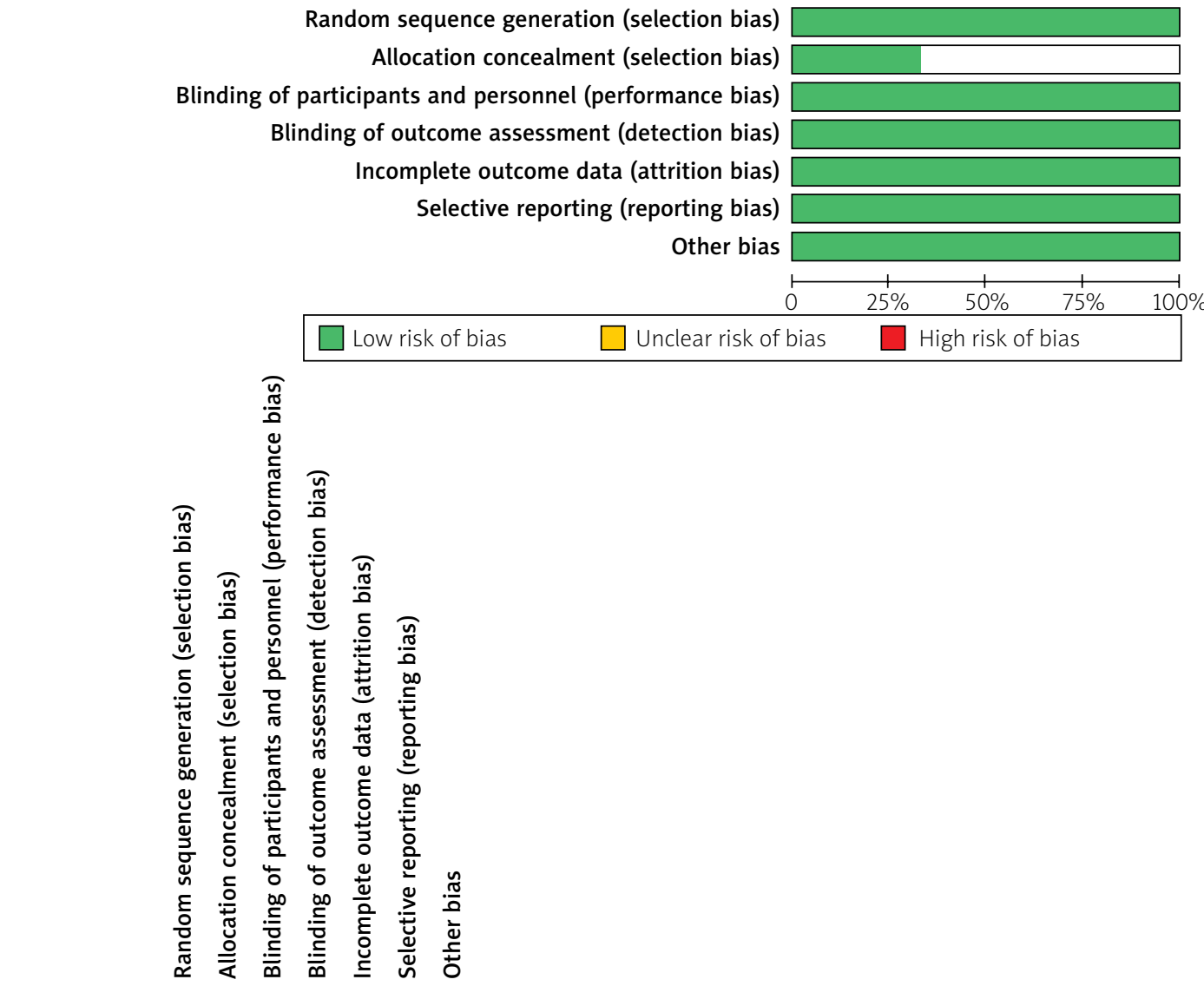

\begin{tabular}{|c|c|c|c|c|c|c|c|}
\hline Ajmera 2019 & + & + & + & + & + & + & + \\
\hline & + & & + & + & + & + & + \\
\hline 014 & + & & + & + & + & + & + \\
\hline
\end{tabular}

Figure 2. The risk of bias graph and summary of the included studies

gorized as "low risk" of bias, while the other 2 studies $[20,26]$ did not report sufficient details about concealment, so they were put to unclear risk of bias. Regarding blinding of outcome assessment, 2 studies [25, 26] reported adequate blinding, so they were put to low risk of bias, while the other study did not report enough details regarding blinding of assessors, so it was categorized as "unclear risk". A detailed illustration of the risk of bias of included trials is summarized in Figure 2.

\section{Analysis of outcomes}

\section{Alanine aminotransferase}

All studies $[20,25,26]$ reported ALT outcome. The overall mean difference did not show any significant difference between both groups (MD = $3.92[-21.20$, 29.04], $p=0.76$ ). Pooled analysis was heterogeneous; $p=0.03 ; 1^{2}=88 \%$, as shown in Figure $3 \mathrm{~A}$. We solved heterogeneity by excluding Levin et al. [26]; $p=0.88$; $1^{2}=0 \%$. The overall analysis after solving heterogeneity showed a significant increase in ALT levels in the Aram- chol group (MD $=15.94[2.83,29.06], p=0.02)$ as show in Figure $3 \mathrm{~B}$.

\section{Aspartate aminotransferase}

Two studies $[20,25]$ reported AST outcome. The AST level was significantly higher in the Aramchol group $(M D=11.04$ [4.91, 17.16], $p=0.04)$. Data was homogeneous; $p=0.77 ; l^{2}=0 \%$, as shown in Figure 4 .

\section{Alkaline phosphatase}

Two studies $[20,25]$ reported the AP outcome. The overall mean difference did not show any difference between both groups (MD $=-0.59[-8.85,7.67], p=0.89$ ). Data were homogeneous; $p=0.52 ; 1^{2}=0 \%$, as shown in Figure 5.

\section{$\mathrm{Hb}_{1 \mathrm{c}}$}

All studies $[20,25,26]$ reported the $\mathrm{HbA}_{1 c}$ outcome. The combined analysis showed no difference between either group ( $M D=-0.11[-0.32,0.10], p=0.29)$. Pooled 


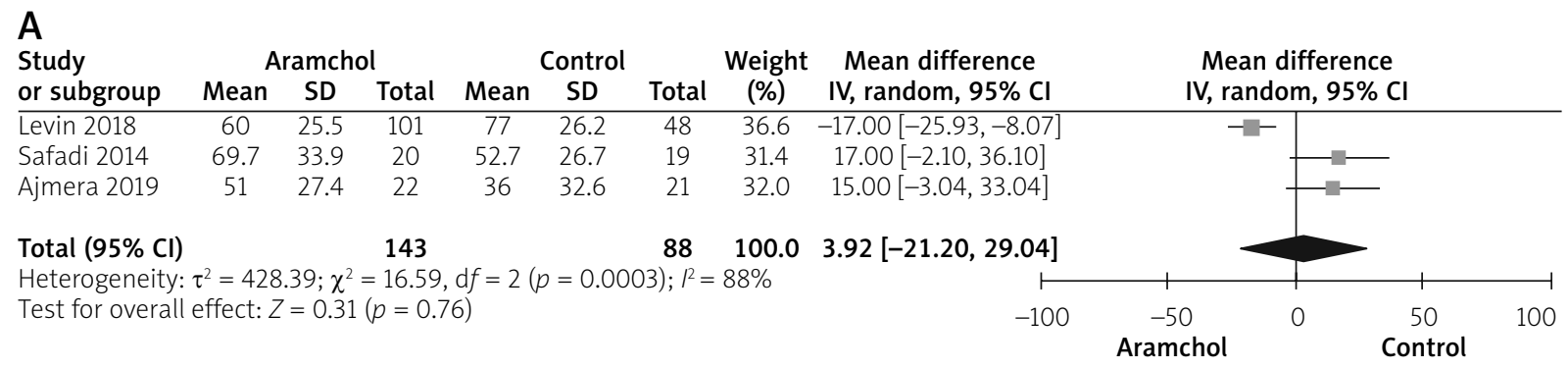

B

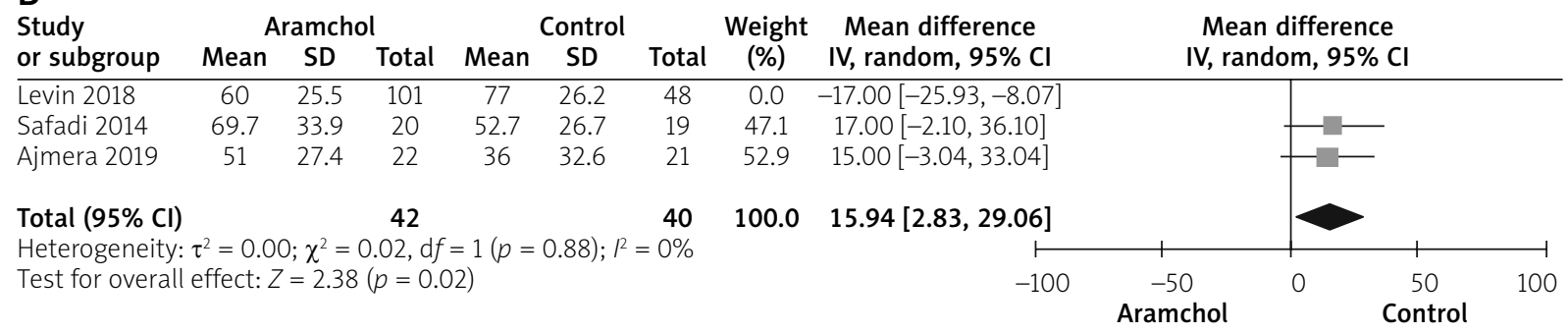

Figure 3. The analysis of ALT outcome

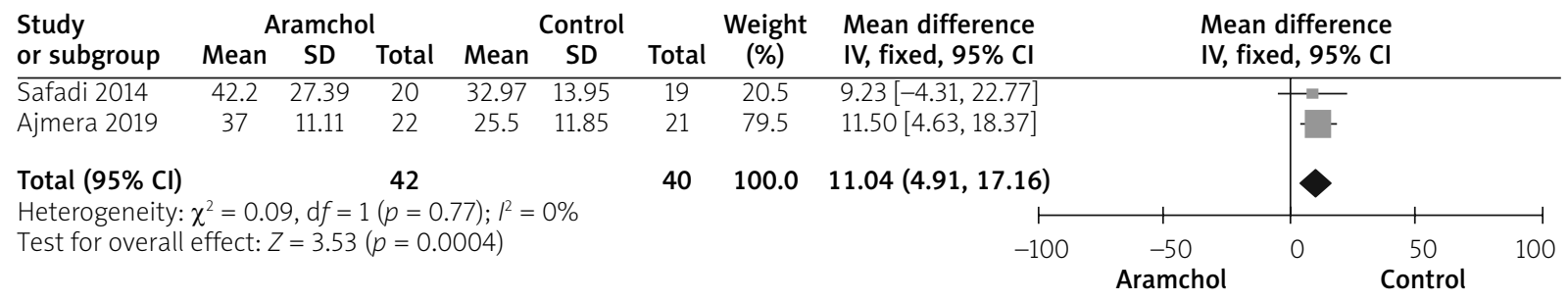

Figure 4. The analysis of AST outcome

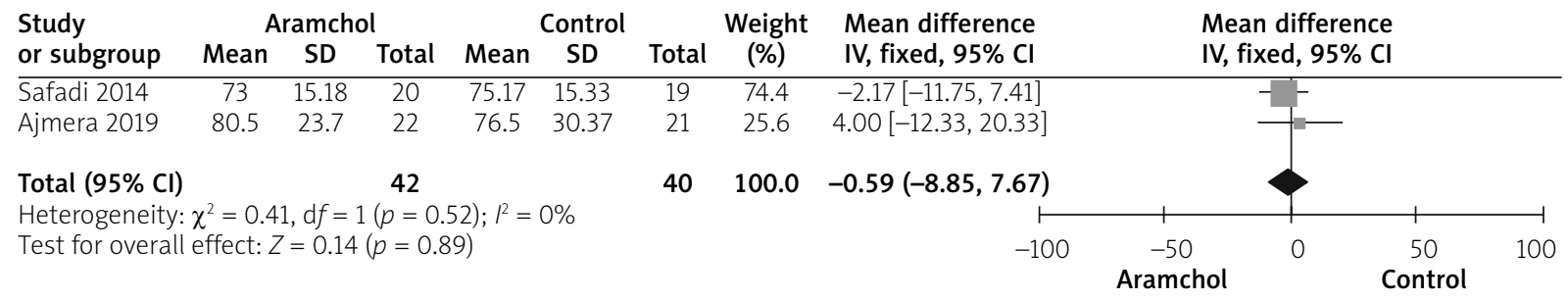

Figure 5. The analysis of AP outcome

analysis was heterogeneous; $p=0.06 ; 1^{2}=64 \%$, as shown in Figure 6 A. We solved heterogeneity by leaving Safadi et al. [20] out; $p=0.85 ; l^{2}=0 \%$. The combined mean difference after solving heterogeneity favoured the Aramchol group (MD $=-0.22[-0.36,-0.08], p=$ 0.02), as shown in Figure 6 B.

\section{Total cholesterol}

Two studies [20,25] reported TC. The overall analysis did not show any significant difference between both groups ( $M D=14.25[-6.26,34.77], p=0.17)$. Pooled analysis was homogeneous; $p=0.89 ; l^{2}=0 \%$, as shown in Figure 7.

\section{Triglyceride}

Two studies [20, 25] reported triglyceride. Pooled analysis did not favour any group over the other (MD = 2.29 [-39.30, 43.87], $p=0.91$ ). Data were homogenous; $p=0.55 ; r^{2}=0 \%$, as shown in Figure 8 .

\section{$H O M A-I R$}

Two studies $[20,25]$ reported HOMA outcome. The overall analysis did not show any significant difference between both groups ( $M D=-0.11$ [-1.58, 1.37], $p=0.89$ ). Pooled analysis was homogeneous; $p=0.39$; $P^{2}=0 \%$, as shown in Figure 9 . 


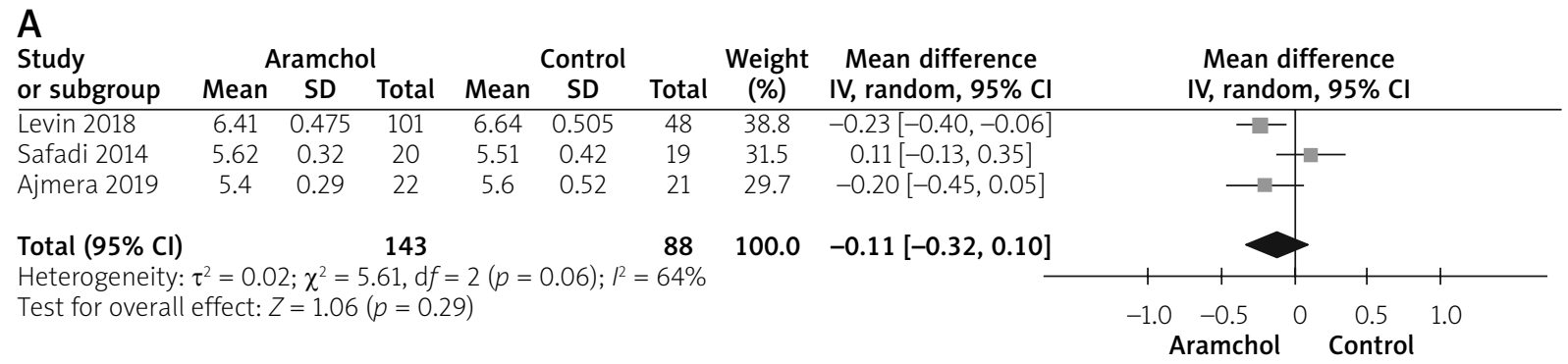

B

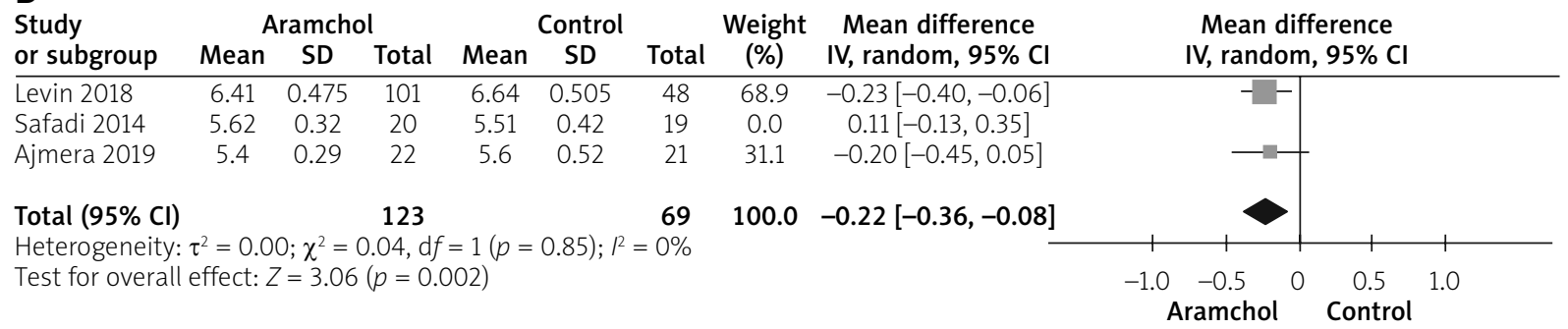

Figure 6. The analysis of $\mathrm{HbA}_{1 \mathrm{c}}$ outcome

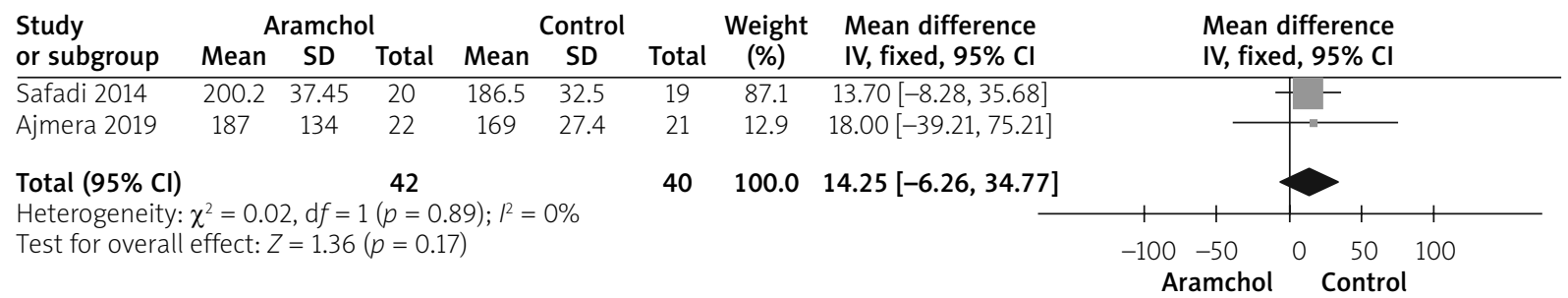

Figure 7. The analysis of total cholesterol outcome

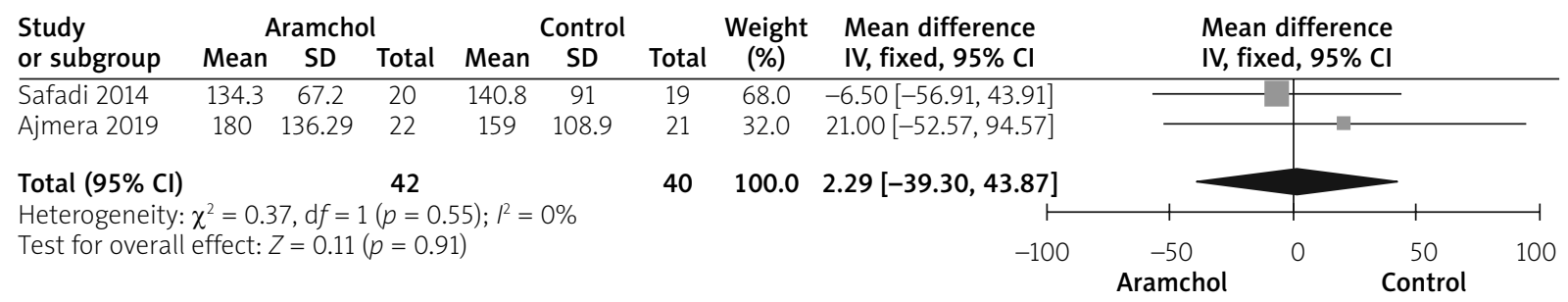

Figure 8. The analysis of TAG outcome

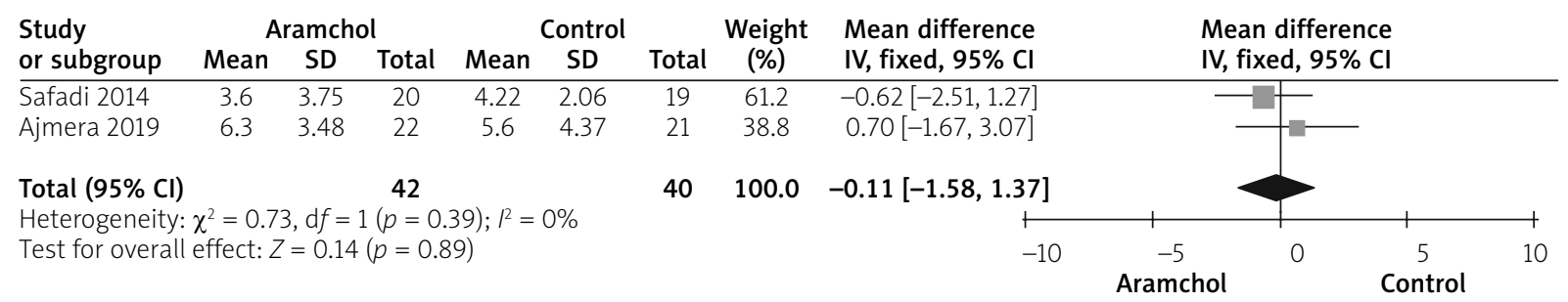

Figure 9. The analysis of HOMA outcome 


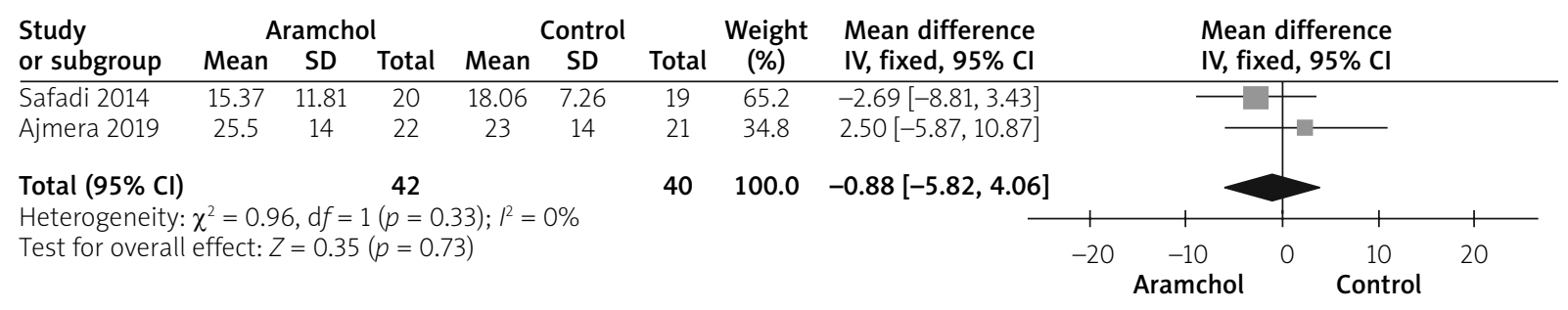

Figure 10. The analysis of insulin outcome

\section{Insulin levels}

Insulin was reported by 2 studies [20, 25]. The overall mean difference did not show any significant difference between both groups (MD $=-0.88[-5.82,4.06]$, $p=0.73$ ). Data were homogeneous; $p=0.33 ; l^{2}=0 \%$, as shown in Figure 10 (Tables II, III).

\section{Discussion}

In this meta-analysis, we included 231 patients with NAFLD treated with Aramchol from 3 randomized controlled trials. We found that Aramchol did not show any significant reduction in AST and ALT levels. The results did not also show any significant difference between Aramchol and placebo regarding the level of plasma cholesterol, triglyceride, $\mathrm{AP}, \mathrm{HbA}_{1 \mathrm{c}}$, and $\mathrm{HOMA}$ score.

ARRIVE [25] is the first randomized, double-blind, placebo-controlled trial that used an advanced MRIbased measure of body composition, including total visceral adipose tissue (VAT) and vibration-controlled transient elastography (VCTE) before and after the treatment. The trial found that Aramchol was not associated with a significant reduction in hepatic fat, ami- notransferases, or liver stiffness compared with placebo. On the other hand, Aramchol may lead to a significant reduction in ALT level, especially among obese patients with HIV-associated NAFLD. Throughout the trial, Aramchol was safe and well-tolerated with no significant side effects and a similar safety profile to placebo. The ARRIVE trial did not include the histological examination of the liver as a diagnostic measure, which may, in turn, limit the ability to detect the early improvement in disease activity, especially those related to the improvement in ALT. Aramchol is a potent oral hepatic stearoyl-CoA desaturase 1 (SCD1) modulator. It belongs to medications targeting the primary metabolic process; specifically, the subgroup of inhibiting de-novo lipogenesis $[27,28]$. SCD-1 is the rate-limiting enzyme in hepatic lipogenesis. Aramchol causes downregulation of SCD-1, which converts saturated fatty acids into monounsaturated fatty acids [29]. SCD-1 downregulation results in a reduction in hepatic lipogenesis, adiposity, liver triglycerides, and obesity resistance [30]. In addition, it acts on (ABCA1) transporter, a cholesterol efflux pump, producing an antiatherogenic effect [31].

Table II. Baseline values of ALT, AST, alkaline phosphatase, HOMA-IR, and $\mathrm{HbA}_{1 \mathrm{c}}$

\begin{tabular}{|c|c|c|c|c|c|c|c|c|c|c|}
\hline \multirow[t]{2}{*}{ Study } & \multicolumn{2}{|c|}{ ALT [IU/I] } & \multicolumn{2}{|c|}{ AST [IU/I] } & \multicolumn{2}{|c|}{$\begin{array}{c}\text { Alkaline phosphatase } \\
\text { [IU/I] }\end{array}$} & \multicolumn{2}{|c|}{ HOMA-IR } & \multicolumn{2}{|c|}{$\mathrm{HbA}_{1 \mathrm{c}}(\%)$} \\
\hline & Aramchol & Control & Aramchol & Control & Aramchol & Control & Aramchol & Control & Aramchol & Control \\
\hline $\begin{array}{l}\text { Ajmera } \\
2019\end{array}$ & $58(39.25)$ & $43(19.26)$ & $40.5(21.48)$ & $28.5(18.51)$ & 77 (22.96) & $79.5(31.11)$ & $7.2(3.4)$ & $6.2(6.07)$ & $5.4(.29)$ & $5.6(0.52)$ \\
\hline Levin 2018 & $68.1(48.3)$ & $67.7(47.5)$ & NR & NR & NR & NR & NR & NR & $6.5(0.9)$ & $6.5(1)$ \\
\hline $\begin{array}{l}\text { Safadi } \\
2014\end{array}$ & $78.3(41.6)$ & $63.1(31.2)$ & $47.8(33.8)$ & $38.8(16.4)$ & 75.7 (17.8) & $73.5(24.5)$ & $4.4(3.7)$ & $4.4(2.7)$ & $5.5(0.3)$ & $5.4(0.5)$ \\
\hline
\end{tabular}

Data represented as mean (SD). ALT - alanine aminotransferase, AST - aspartate aminotransferase, NR - not reported.

Table III. Baseline values of total cholesterol, HDL, LDL, and insulin

\begin{tabular}{lcccccccc} 
Study & \multicolumn{2}{c}{ Total cholesterol $[\mathrm{mg} / \mathrm{dl}]$} & \multicolumn{2}{c}{ HDL $[\mathrm{mg} / \mathrm{dl}]$} & \multicolumn{2}{c}{ LDL $[\mathrm{mg} / \mathrm{dl}]$} & \multicolumn{2}{c}{ Insulin [mIU/ml] } \\
\cline { 2 - 9 } & Aramchol & Control & Aramchol & Control & Aramchol & Control & Aramchol & Control \\
\hline Ajmera 2019 & $177(42.96)$ & $179(40)$ & NR & NR & $110(33.3)$ & $96(20)$ & $29.5(12.59)$ & $24.5(18.5)$ \\
\hline Levin 2018 & NR & NR & NR & NR & NR & NR & NR & NR \\
\hline Safadi 2014 & $193.6(43.5)$ & $187(40.7)$ & $43.5(19.8)$ & $41.6(7.6)$ & $122.3(33.6)$ & $116.2(34.4)$ & $18.2(11.3)$ & $18.3(9.8)$
\end{tabular}

Data represented as mean (SD). HDL - high-density lipoprotein, $L D L$-low-density lipoprotein, NR-not reported. 
In animal models, a trial by Iruarrizaga-Lejarreta et al. [19] found that Aramchol played a significant role in the improvement of steatohepatitis and fibrosis. This improvement was explained by the effect of the drug on SCD- 1 and the use of an alternative trans-sulphuration pathway maintaining cellular redox homeostasis.

A trial by Safadi et al. [20] found that Aramchol was associated with a significant dose-dependent improvement in liver function. Patients treated with high-dose Aramchol had a significant reduction of symptoms related to liver cell failure compared with placebo. It was also found that daily administration of $300 \mathrm{mg}$ Aramchol was tolerable and safe and causes a significant reduction in reduces liver fat content in patients with NAFLD. However, the short therapeutic protocol of the trial represented a strong limitation to estimate the clinical effect of Aramchol on liver fat.

In a 1-year ARREST study [26], Aramchol was found to reduce liver fat and improve the biochemical markers of the liver. The drug was also found to reduce liver fibrosis in a dose-response pattern. Administration of Aramchol $600 \mathrm{mg}$ demonstrated higher rates of NASH resolution and fibrosis reduction with excellent safety outcomes. The major strengths of our analysis were that we included only clinical trials, which is important to ensure the strongest evidence according to GRADE. Another strength is that all the included studies were at low risk of bias in general. We tried to solve any inconsistency among studies using appropriate methodologies reported by Cochrane's handbook [32].

The heterogeneity in some outcomes was the major limitation; however, we tried to solve the heterogeneity by subgroup analysis. Another limitation was the small sample size and a low number of published clinical trials; therefore, we still recommend more trials to combine Aramchol with other medications or to use higher doses below the safety margin of the drug.

\section{Conclusions}

Aramchol was a safe and tolerable drug for use in patients with NAFLD. However, it was not superior to the placebo at reducing the biochemical liver markers.

\section{Conflict of interest}

The authors declare no conflict of interest.

\section{References}

1. Ludwig J, Viggiano TR, McGill DB, Oh BJ. Non-alcoholic steatohepatitis: Mayo Clinic experiences with a hitherto unnamed disease. Mayo Clin Proc 1980; 55: 434-8.

2. Cohen JC, Horton JD, Hobbs $\mathrm{HH}$. Human fatty liver disease: old questions and new insights. Science 2011; 332: 1519-23.
3. Vernon G, Baranova A, Younossi ZM. The epidemiology and natural history of non-alcoholic fatty liver disease and non-alcoholic steatohepatitis in adults. Aliment Pharmacol Ther 2011; 34: 274-85.

4. Boza C, Riquelme A, Ibañez L, et al. Predictors of non-alcoholic steatohepatitis (NASH) in obese patients undergoing gastric bypass. Obes Surg 2005; 15: 1148-53.

5. Bedogni G, Miglioli L, Masutti F, et al. Prevalence of and risk factors for non-alcoholic fatty liver disease: the dionysos nutrition and liver study. Hepatology 2005; 42: 44-52.

6. Younossi ZM, Stepanova M, Afendy $M$, et al. Changes in the prevalence of the most common causes of chronic liver diseases in the United States from 1988 to 2008. Clin Gastroenterol Hepatol 2011; 9: 524-30.

7. Trefts E, Gannon M, Wasserman DH. The liver. Curr Biol 2017; 27: R1147-51.

8. Lee RG. Non-alcoholic steatohepatitis: a study of 49 patients. Hum Pathol 1989; 20: 594-8.

9. Sanyal AJ, Campbell-Sargent C, Mirshahi F, et al. Non-alcoholic steatohepatitis: Association of insulin resistance and mitochondrial abnormalities. Gastroenterology 2001; 120: 118392.

10. Donnelly KL, Smith CI, Schwarzenberg SJ, et al. Sources of fatty acids stored in liver and secreted via lipoproteins in patients with non-alcoholic fatty liver disease. J Clin Invest 2005; 115 : 1343-51.

11. Lambert JE, Ramos-Roman MA, Browning JD, Parks EJ. Increased de novo lipogenesis is a distinct characteristic of individuals with non-alcoholic fatty liver disease. Gastroenterology 2014; 146: 726-35.

12. Ratziu V. Novel pharmacotherapy options for NASH. Digest Dis Sci 2016; 61: 1398-405.

13. Lassailly G, Caïazzo R, Pattou F, Mathurin P. Bariatric surgery for curing NASH in the morbidly obese? J Hepatol 2013; 58: 1249-51.

14. Dobrzyn P, Dobrzyn A, Miyazaki M, et al. Stearoyl-CoA desaturase 1 deficiency increases fatty acid oxidation by activating AMP-activated protein kinase in liver. Proc Natl Acad Sci USA 2004; 101: 6409-14.

15. Dobrzyn A, Ntambi JM. Stearoyl-CoA desaturase as a new drug target for obesity treatment. Obesity Rev 2005; 6: 169-74.

16. Konikoff FM, Gilat T. Effects of fatty acid bile acid conjugates (FABACS) on biliary lithogenesis: potential consequences for non-surgical treatment of gallstones. Curr Drug Targets Immune Endocr Metabol Disord 2005; 5: 171-5.

17. Leikin-Frenkel A, Goldiner I, Leikin-Gobbi D, et al. treatment of preestablished diet-induced fatty liver by oral fatty acid-bile acid conjugates in rodents. Eur J Gastroenterol Hepatol 2008; 20: 1205-13.

18. Leikin-Frenkel A, Gonen A, Shaish A, et al. Fatty acid bile acid conjugate inhibits hepatic stearoyl coenzyme a desaturase and is non-atherogenic. Arch Med Res 2010; 41: 397-404.

19. Iruarrizaga-Lejarreta M, Varela-Rey M, Fernández-Ramos D, et al. Role of aramchol in steatohepatitis and fibrosis in mice. Hepatol Commun 2017; 1: 911-27.

20. Safadi R, Konikoff FM, Mahamid M, et al. The fatty acid-bile acid conjugate aramchol reduces liver fat content in patients 
with nonalcoholic fatty liver disease. Clin Gastroenterol Hepatol 2014; 12: 2085-91.e1.

21. Moher D, Liberati A, Tetzlaff J, et al. Preferred reporting items for systematic reviews and meta-analyses: the PRISMA statement. J Chinese Integr Med 2009; 7: 889-96.

22. Higgins JPT, Green S. Cochrane Handbook for Systematic Reviews of Interventions: Cochrane Book Series. Vol. Version 5. 2008.

23. Higgins JPT, Thompson SG, Deeks JJ, Altman DG. Measuring inconsistency in meta-analyses. Br Med J 2003; 327: 557-60.

24. Higgins JP, Altman DG. Assessing Risk of Bias in Included Studies. In: Cochrane Handbook for Systematic Reviews of Interventions: Cochrane Book Series. 2008.

25. Ajmera VH, Cachay E, Ramers C, et al. MRI assessment of treatment response in HIV-associated NAFLD: a randomized trial of a stearoyl-coenzyme-A-desaturase-1 inhibitor (ARRIVE Trial). Hepatology 2019; 70: 1531-45.

26. Levin J, Ratziu V, de Guevara L, et al. One-year results of the Global Phase $2 b$ randomized placebo-controlled ARREST Trial of Aramchol, a Stearoyl CoA Desaturasemodulator in NASH patients. Liver Meet [Internet]. 2018; Available from: http:// www.natap.org/2018/AASLD/AASLD_222.htm.

27. Higgins J, Altman D. Assessing risk of bias. In: Cochrane Handbook for Systematic Reviews of Interventions: Cochrane Book Series. 2008.

28. Flowers MT, Miyazaki M, Liu X, Ntambi JM. Probing the role of stearoyl-CoA desaturase-1 in hepatic insulin resistance. J Clin Investig 2006; 116: 1478-81.

29. Dobrzyn P, Sampath H, Dobrzyn A, et al. Loss of stearoyl-CoA desaturase 1 inhibits fatty acid oxidation and increases glucose utilization in the heart. Am J Physiol Endocrinol Metab 2008; 294: E357-64.

30. ALJohani AM, Syed DN, Ntambi JM. Insights into stearoyl-CoA desaturase-1 regulation of systemic metabolism. Trends Endocrinol Metab 2017; 28: 831-42.

31. Goldiner I, Van Der Velde AE, Vandenberghe KE, et al. ABCA1-dependent but apoA-l-independent cholesterol efflux mediated by fatty acid-bile acid conjugates (FABACs). Biochem J 2006; 396: 529-36.

32. Higgins JPT, Green S (eds.). Cochrane Handbook for systematic reviews of interventions version 5.1.0 [updated March 2011] The Cochrane Collaboration 2011.

Received: 21.12.2021

Accepted: 30.12 .2021 